\title{
Gluten and Neuroimmunology. Rare association with Myasthenia Gravis and Literature Review
}

\author{
Francisco Oliveira ${ }^{1}$ \\ Vinicius Schoeps ${ }^{1}$ \\ Wilson Sanvito ${ }^{1}$ \\ Berenice Valerio ${ }^{2}$
}

1. Department of Neurology, Santa Casa at São Paulo-SP, Brasil. Ambulatory of Neurological manifestations of systemic diseases. 2. Ambulatory of neuromuscular diseases.

\section{SUMMARY}

As the celiac disease (CD), the non-celiac gluten sensitivity (NCGS) has also been associated with several autoimmune manifestations. It is rarely associated with myasthenia gravis (MG). This paper shall introduce the case of a young female patient, initially presenting a peripheral neuropathy framework. During clinical and neurological follow-up, she began to present symptoms of various immune-mediated morbidities. Diseases related to gluten represent a clinical spectrum of manifestations with a trigger in common, the ingestion of gluten. CD is the most well-known and serious disease of the spectrum, also called gluten-sensitive enteropathy. The NCGS is diagnosed from clinical evidence of improvement in symptoms followed by a Gluten Free Diet (GFD) in patients without signs of enteropathy in duodenal biopsy. There are indications that, although rare, with a prevalence of 1 in 5000 , myasthenia gravis (MG) may occur more often when CD is also present. Between 13 to $22 \%$ of the patients with MG have a second autoimmune disorder. However, it is often associated with dermatomyositis or polymyositis, lupus erythematosussystemic lupus erythematosus, Addison's disease, Guillain-Barré syndrome and juvenile rheumatoid arthritis. Thus, the symptoms of neuromuscular junction involvement may give a diagnostic evidence of this rare association.

KEYWORDS: Celiac disease. Glutens. Myasthenia gravis. Autoimmune diseases.

\section{INTRODUCTION}

Celiac disease (CD) is an autoimmune disorder accompanied by a variety of neurological manifestations. Within its clinical spectrum, there is the non-celiac gluten sensitivity (NCGS), which presents clinical symptoms similar to those of patients with $\mathrm{CD}$, but without histopathological evidence of enteropathy in duodenal biopsy. Both disorders present extra-intestinal autoimmune manifestations. However, it is rarely associated with myasthenia gravis (MG). In this article, we present a case of an NCGS patient, who developed peripheral neuropathy associated to vitamin B12 deficiency, ataxia related to gluten, epilepsy and later Myasthenia Gravis.

\section{CASE}

S.V.B., is a 34-year-old female, born in São Paulo, with an incomplete bachelor's degree in Psychology, and she is a nun. She started presenting distal paresthesia, in all four limbs accompanied by chronic diarrhea, when she was 20 years old, being diagnosed with NCGS. After a year, she started presenting axial and appendicular ataxia in addition to generalized tonic-clonic seizures, and right tactile hypoesthesia. In the Department of Neurology, it was conducted the hypothesis of ataxia related to gluten, epilepsy and secondary peripheral neuropathy due to vitamin B12 deficiency or NCGS. Brain magnetic resonance imaging (MRI) found a cerebellar atrophy, which was

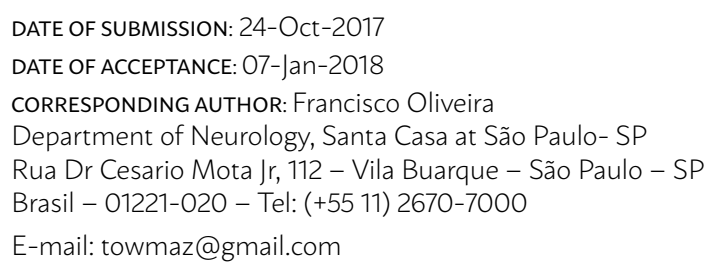


consistent with the clinical picture. After introducing gluten-free diet (GFD) she had partial improvement of neurological and gastrointestinal symptoms. A year ago, she started to present a progressive asthenia, tetraparesia predominantly proximal, dyspnea, dysarthria, diplopia, dysphonia and bilateral palpebral ptosis. Good therapeutic response with pyridostigmine. She was diagnosed with Myasthenia Gravis. She was already using prednisone and azathioprine due to gastrointestinal alterations.

\section{DISCUSSION}

Gluten related diseases represent a clinical spectrum of manifestations with a common factor, ingestion of gluten. ${ }^{1}$ The most well-known and serious disease of the spectrum is $\mathrm{CD}$, also called gluten-sensitive enteropathy. Diagnosis of NCGS is made from a clinical evidence of improvement in symptoms followed by a DLG in patients without signs of enteropathy in duodenal biopsy. ${ }^{2}$ Both DC as SGNC are responsive to DLG, suggesting a similar physio/ pathophysiologic mechanisms. ${ }^{3}$ The neurological manifestations of DC and SGNC are similar and occur in up to $10 \%$ of patients. ${ }^{3,4}$ Among the most common are peripheral neuropathy in $30 \%$ of the cases and cerebellar ataxia in $41 \%$ (see graph), both probably related to autoimmune involvement. ${ }^{2}$

In this context, peripheral neuropathy is predominantly distal, sensory motor and slowly progressive and symmetrical. It does not have a unique pathophysiological mechanism defined, but it is believed that the lack of vitamins and nutrients is not the sole cause, and may even be a slightly cause in most cases, since most of the patients do not present a serious enteropathy associated with a malabsorption syndrome. ${ }^{5}$ In the case presented, there is vitamin B12 deficiency, despite enteropathy absence, that could have contributed to the onset of neuropathy. It is speculated that there must have an overlap of causes associated with pernicious anemia due to refractivity of the case and development of various immune- mediated morbidities. In addition, according to literature, a free malabsorption syndrome occurs in only $30 \%$ of untreated patients and it is not severe in patients undergoing regular and good monitoring therapeutic compliance (like the case of our patient). In general, it is a belief that neuropathy must be immune-mediated just as ataxia related to gluten.

Ataxia usually presents insidiously, with an aver- age age of 53 years, with cases reported in pediatric patients. All affected patients have gait ataxia and most predominantly in the lower limbs. Less than $10 \%$ will present any gastrointestinal symptom, only $40 \%$ will have evidence of enteropathy. Thus, ataxia has similar evolution to dermatitis herpetiformis, which is a skin disease, triggered by gluten, wherein the gastrointestinal symptoms are not very prominent even in the presence of enteropathy.

In imaging studies of the central nervous system, $60 \%$ of patients have cerebellar atrophy, but it can be demonstrated abnormalities to spectroscopy with reduced N-acetyl aspartate / creatinine ratio, primarily affecting the cerebellar vermis in all of them. Postmortem studies of these patients reveal a focal degeneration of Purkinge cells through the cerebellar cortex associated with astrocytic gliosis and diffuse infiltration of T-cells in the white matter. Experimental evidences suggest a cross-reactivity between epitopes of Purkinge cells (and other of the granular layer) and gluten peptides. Such cross-reactivity has been attributed to anti-gliadin antibodies, but with increased evidence of associated involvement of anti-transglutaminase antibodies.

Transglutaminase 6 (TG6), expressed in brain tissue, is described as a target antigen in these patients. Anti TG6 autoantibodies are discarded after introducing gluten-free diet. These probably penetrate the central nervous system by reacting with the cell and granular Purkinje after disruption of the blood-brain barrier triggered by perivascular deposits of IgA. ${ }^{6}$ The loss of Purkinje cells after prolonged exposure to gluten is irreversible, so that only the early diagnosis and treatment are able to result in stabilization or clinical improvement. ${ }^{6,7}$

There are indications that, although rare, with a prevalence of 1 in 5000, myasthenia gravis (MG) may occur more frequently when $\mathrm{CD}$ is also present. ${ }^{3}$ The symptoms of neuromuscular junction involvement can give a diagnostic clue of this rare association., ${ }^{3,8}$ About 13 to $22 \%$ of MG patients have a second autoimmune disease. However, it is more often associated with Dermatomyositis or polymyositis, lupus erythematosus, Addison disease, Guillain-Barré syndrome and juvenile rheumatoid arthritis. ${ }^{8}$ This combination does not change when we conducted studies for patients with or without thymoma. It is worth mentioning that among the three diseases most associated with MG: Dermatomyositis or polymyositis (myopathies Inflammatory Idiopathic), lupus erythematosusSystemic lupus ery- 
thematosus and Adison disease, are all regulated by the haplotype HLA-B8-DR3. More genetic studies are necessary to determine if the combination of these conditions is due to heterogeneity between HLA, interaction between not HLA genes or environmental factors. ${ }^{9}$

In addition to the events listed, there is a close relationship between epilepsy and autoimmune diseases. ${ }^{10,11}$ This association seems to be attributed to the inflammatory part in the underlying disease, shown by consistent histopathological findings in refractory patients undergoing surgery. ${ }^{10,12}$ A retrospective cohort study using insurance companies data with more than 2.5 million patients, found an increase of 3.8 times in patients with autoimmune diseases. The same study identified a 2.5-fold increase in adults with celiac disease and five times in patients with myasthenia gravis. Such evidences will further produce new therapeutic options. ${ }^{10}$

Most studies of neurological complications with enteropathy are generally related to celiac disease and have highlighted the central nervous system involvement. However, it is known that the association with neuropathy, myopathy, myasthenia gravis, Stiff person Syndrome (SPS) and, more rarely with celiac disease exists. ${ }^{2,13}$

Holmes $^{14}$ conducted a descriptive study where the frequency of neuropathy was $0.5 \%$ and $1.3 \%$ in myopathy. In all cases, myopathy was a result of osteomalacia, which is today very unusual in celiac disease, and has a good response to treatment with vitamin $\mathrm{D}$ and gluten-free diet. ${ }^{13,14}$

A recent study showed inflammatory cell infil-

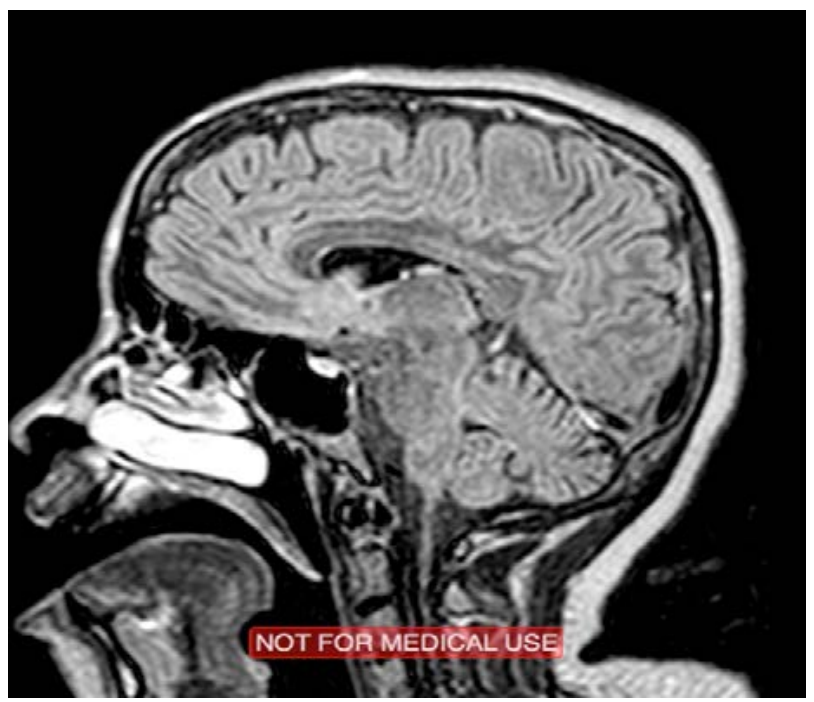

tration in nerve roots of the spinal cord at a patient with celiac disease and polyneuropathy. ${ }^{15}$ The largest series were so far related by Hadjivassiliou et al, ${ }^{16}$ who described six patients with sensorimotor axonal polyneuropathy (of which two with a pure motor neuropathy), a patient with multiple mononeuropathy and a patient with Guillain-Barre syndrome.

The results of the studies show that polyneuropathy associated with celiac disease is axonal and affects both motor and sensitive fibers, with greater pain and heat if compared to the group of control. This finding provides further evidences that peripheral nerve fibers, even unmyelinated fibers, have been affected in patients with celiac disease. ${ }^{13,16}$

\section{CONCLUSION}

There is a significant overlap between autoimmune disorders, reflecting a similar pathological mechanism. In the context of the celiac disease, the role of DLG in the course of autoimmune diseases has been studied and it is related to some neurological improvement or stabilization. However, there is no consensus in literature considering the clinical course of these entities. In this case, we reported a partial clinical improvement of ataxia, neuropathy, and gastrointestinal symptoms, but control of myasthenia is due to the use of immunosuppressive drugs and cholinesterase inhibitors. There are few reports of this association and limited knowledge about them. A better understanding will be crucial for the establishment of more targeted and effective treatments.

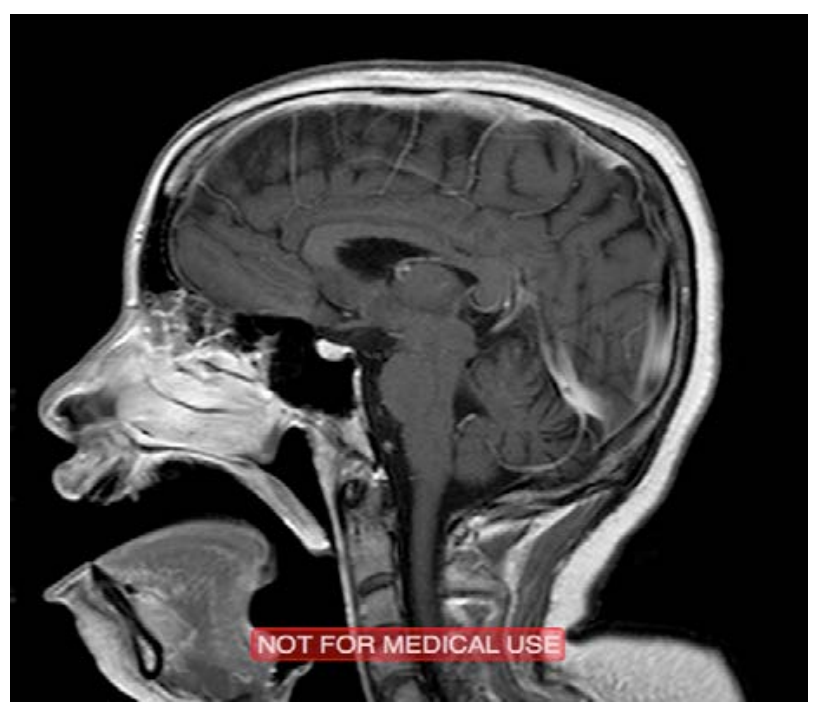

FIGURE 01. MRI Brain: sagittal, respectively, weighted FLAIR and T1 with gadolinium, demonstrating cerebellar atrophy, especially the vermis. 
PALAVRAS-CHAVE: Doença celíaca. Glutens. Miastenia gravis. Doenças autoimunes.

\section{REFERENCES}

1. Catassi C, Elli L, Bonaz B, Bouma G, Carroccio A, Castillejo G, et al. Diagnosis of non-celiac gluten sensitivity (NCGS): the Salerno Experts' criteria. Nutrients. 2015;7(6):4966-77.

2. Hadjivassiliou M, Rao DG, Grìnewald RA, Aeschlimann DP, Sarrigiannis PG, Hoggard N, et al. Neurological dysfunction in coeliac disease and non-coeliac gluten sensitivity. Am J Gastroenterol. 2016;111(4):561-7.

3. Freeman HJ, Gillett HR, Gillett PM, Oger J. Adult celiac disease with acetylcholine receptor antibody positive myasthenia gravis. World J Gastroenterol. 2009;15(38):4741-4.

4. Almeida Menezes M, Ribeiro Cabral VL, Lorena SS, Nucci A, Andrade Santana P, Queiroz Silva C. Rare association of celiac disease with myasthenia gravis in a patient with other immune disorders: a case report. Rev Esp Enferm Dig. 2016;108(9):586-8.

5. Hadjivassiliou M, Grünewald RA, Kandler RH, Chattopadhyay AK, Jarratt A, Sanders DS, et al. Neuropathy associated with gluten sensitivity. | Neurol Neurosurg Psychiatry. 2006;77(11):1262-6.

6. Mitoma H, Adhikari K, Aeschlimann D, Chattopadhyay P, Hadjivassiliou $\mathrm{M}$, Hampe CS, et al. Consensus paper: neuroimmune mechanisms of cerebellar ataxias. Cerebellum. 2016;15(2):213-32.

7. Hadjivassiliou M, Davies-Jones GA, Sanders DS, Grünewald RA. Dietary treatment of gluten ataxia. N Neurol Neurosurg Psychiatry. 2003;74(9):1221-4

8. Nacu A, Andersen JB, Lisnic V, Owe JF, Gilhus NE. Complicating autoimmune diseases in myasthenia gravis: a review. Autoimmunity. 2015:48(6):362-8.
9. Fang $F$, Sveinsson $O$, Thormar G, Granqvist M, Askling |, Lundberg IE, et al. The autoimmune spectrum of myasthenia gravis: a Swedish population-based study. J Intern Med. 2015;277(5):594-604.

10. Ong MS, Kohane IS, Cai T, Gorman MP, Mandl KD. Population-level evidence for an autoimmune etiology of epilepsy. IAMA Neurol. 2014;71(5):569-74

11. Pengiran Tengah DS, Wills AJ, Holmes GK. Neurological complications of coeliac disease. Postgrad Med J. 2002;78(921):393-8.

12. Aronica E, Crino PB. Inflammation in epilepsy: clinical observations. Epilepsia. 2011;52(Suppl 3):26-32.

13. Luostarinen L, Himanen SL, Luostarinen M, Collin P, Pirttilä T. Neuromuscular and sensory disturbances in patients with well treated coeliac disease. | Neurol Neurosurg Psychiatry. 2003;74(4):490-4.

14. Holmes GKT. Neurological and psychiatric complications in coeliac disease. In: Gobbi G, Andermann F, Naccarato S, Banchini G, eds. Epilepsy and other neurological disorders in coeliac disease. London: John Libbey; 1997. p.251-64.

15. Muller AF, Donnelly MT, Smith CM, Grundman MJ, Holmes GK, Toghill P). Neurological complications of celiac disease: a rare but continuing problem. Am | Gastroenterol. 1996;91(7):1430-5.

16. Hadjivassiliou M, Chattopadhyay AK, Davies-Jones GA, Gibson A, Grünewald RA, Lobo Al. Neuromuscular disorder as a presenting feature of coeliac disease. J Neurol Neurosurg Psychiatry. 1997;63(6): $770-5$ 